\title{
SOBRE LA HOSPITALIDAD DE LOS HOSPITALES COMO PARTE FUNDAMENTAL DEL SERVICIO DE SALUD
}

\author{
LUIS GUILLERMO SICHERI MONTEVERDE
}

Universidad Norbert Wiener

En el presente texto se argumenta que además del servicio vinculado a las profesiones de los médicos, enfermeras, químico farmacéuticos y demás personal de salud, que ofrecen su conocimiento para la cura o alivio de enfermedades, es importante la hospitalidad del servicio en clínicas, hospitales y centros de salud. En la perspectiva del paciente, fundamental es curarse; pero también es fundamental sentirse acogido y atendido con cordialidad.

\section{ORIGEN DEL HOSPITAL}

Hay evidencia que indica que a mediados del siglo XIX no era escasa la preocupación recurrente por la calidad de la atención al paciente hospitalizado, como un factor determinante de la recuperación de la salud (Carrera Muñoz 1879).

Pero esta preocupación es aun mucho más antigua; quizá tanto como la de los orígenes del hospital en tanto institución dedicada sistemáticamente a la curación de enfermos. En el marco de las Cruzadas surgieron los Caballeros de San Juan de Jerusalén (u Orden de Malta), llamados hospitalarios, que construyeron los primeros hospitales con el propósito de reparar las energías y los frecuentes problemas de salud que sufrían los peregrinos y comerciantes que recorrían los diferentes caminos que llevaban a Jerusalén o sus diversos destinos intermedios. Se puede documentar en la vertiente francesa de los Pirineos el hospital Saint-Jean de Joeu, en el valle de Luchon, hacia 1200 y, a partir de él en territorio español, el hospital que luego se conoció como hospital de Benasque.

En 1172, el rey Alfonso II de Aragón donó a los hospitalarios un terreno de pastos - rico en aguas sulfurosas- cerca al puerto de Gorgutes, en Huesca. Allí se levantó, seguramente ya en el siglo XIII, lo que sería el primer hospital en España. Su propósito principal -igual que el de Joeu y otros posteriores- era atender el alojamiento y la alimentación de los viajeros de entonces. A eso se agregaba la atención curativa de enfermos y heridos. En el mismo entorno territorial se fueron construyendo sucesivos hospitales, hasta que -a fines del siglo XVII- se inauguró formalmente el hospital de Benasque, en actual investigación arqueológica.

Este tiene alta significación histórica porque existen registros escritos de la función del hospital, en virtud a las obligaciones que en 1847 impuso el ayuntamiento del puerto al concesionario: deberá atender con agrado el servicio; cada ańo deberá blanquear el recinto y 
cambiar los cristales rotos; deberá cobrar 2.50 pesetas por pernocte al nativo de Benasque y 5 pesetas al forastero; deberá ayudar a buscar los cadáveres; no deberá tener más de cien cabezas de ganado lanar para el establecimiento; y deberá tener expuesta una hoja con las normas de establecimiento.

De lo referido se entiende que la función hospitalaria del hospital corresponde a la misma matriz histórica que la función hospitalaria del mesón, de la posada, del albergue, del hotel, del alojamiento en general.

\section{LA FUNCIÓN CURATIVA DEL HOSPITAL}

Lo que importa es la curación; pero hay un aspecto psicológico y social vinculado al servicio que ofrecen las instituciones particulares, así como las instituciones del Estado, que supone que no son suficientes los servicios de médicos, enfermeras y medicinas.

En el plano anímico, es necesario que el paciente se sienta bien para tener ganas de curarse. Y las ganas de curarse eventualmente son determinantes para la salud. Sin embargo, ingresar al hospital puede ser un drama: el hecho del requerimiento de hospitalización es evidencia de la gravedad de la enfermedad; solo se ingresa si hay gravedad o necesidad. Esto supone al paciente una aprensión o angustia naturales, lo que incrementa a nivel psicosomático la gravedad de la enfermedad. Ahora bien, esto puede limitarse o frenarse y decidir la diferencia entre curarse y no curarse. Comprender que el trato al paciente es principalísimo es dar inicio a una actitud sanadora. Ignorar que el paciente requiere el mejor trato posible es dar inicio al deterioro o, peor, a la agonía del paciente.
La atención hospitalaria requiere un protocolo de servicio que se extiende mucho más allá de las ciencias de la salud y que, más bien, se vincula con las ciencias de la hospitalidad. La hospitalidad es consustancial al hospital. El paciente es un huésped especial, que requiere la más dedicada atención; porque esta puede determinar su voluntad de curarse o no. Al respecto, se propone aquí que una parte sustantiva de la misión de la academia sea el desarrollo de la competencia profesional de la conquista de la voluntad del paciente para aportar a su propia curación.

\section{LA EXPECTATIVA DE UN PACIENTE}

Leonard Berry -uno de los más importantes tratadistas del marketing de los servicios- establece que las expectativas de cualquier persona están afectadas por modificadores (Berry y Parasuraman 1993). Una emergencia, por ejemplo, incrementa las expectativas. Un paciente en un hospital o en una clínica modifica sus expectativas. Puede ser que se vuelva particularmente exigente o puede ser que experimente resignación. En cualquier caso, sus expectativas no serán habituales.

Los profesionales que tratan con pacientes hospitalizados deben comprender que están atendiendo a personas cuyas expectativas se han modificado por el solo hecho de estar hospitalizadas. Por tanto, el servicio que requieren es diferencial. Así, quienes atienden a los pacientes hospitalizados deben ser tan buenos o mejores que quienes prestan servicios a huéspedes en hoteles u otros establecimientos de alojamiento. La calidad de los servicios referida a los pacientes de un hospital comporta una calidad específica: consiste en una atención que 
debe ser mejor que aquella que se brinda a personas sanas en cualquier empresa de hospedaje.

Un hospital o una clínica deben ser un escenario de excelencia del servicio; y la excelencia no debe remitirse solo a los médicos o a las enfermeras, que indudablemente deben ser excelentes, sino a todo el personal. El problema es que al generalizar a veces se olvida el desarrollo de las competencias específicas del personal de salud. Si un paciente requiere atención óptima, los médicos y enfermeras, además de los empleados administrativos, deben exhibir competencias de buen trato y eficiencia en el ingreso, permanencia y salida de la experiencia hospitalaria.

Un internamiento tardío genera disconformidad y estrés consecuente. Una salida demorada causa desagrado y molestia innecesaria. Un trato despectivo durante el período de hospitalización ocasiona frustración o indignación contraproducentes. El personal hospitalario debe saber que la hospitalidad es parte del núcleo del servicio que brinda. Los gerentes o administradores de las clínicas y hospitales deben saberlo con mayor razón.

\section{LA ADECUADA INFORMACIÓN}

Aurora Valls y Concepción Rodríguez han realizado un estudio sobre el derecho a la información del paciente, según estándares europeos, desde el punto de vista del rol del bibliotecario, entendido este como consultor del acceso a la información por parte del paciente (2008). Esta perspectiva evidencia que la información dada al paciente no es, en sentido estricto, un tema médico o, al menos, no solo un asunto médico; más aun en el presente, cuando la información accesible ame- ritaría una guía adecuada por su gigantesco volumen. Las autoras identifican diferentes marcos de referencia: psicológico, legal y ético principalmente; si bien el segundo puede no ser aplicado sino por analogía a otras construcciones normativas, los marcos psicológico y deontológico son plenamente replicables en cualquier otro espacio territorial y social.

Por otro lado, Valls y Rodríguez toman una afirmación contenida en la cuenta Roth IRA de 1978, que señala que la información pertinente al paciente reduce los costos de la curación y del aseguramiento, visto que colabora con ella al tener información adecuada y, por tanto, un impacto económico significativo.

El estudio, entre otras cosas -recuerda la Ottawa Charter for Health Promotion de la OMS del año 2000-, considera que la salud no solo es la ausencia de la enfermedad, sino también la adquisición de hábitos saludables de vida. En consecuencia, la información adecuada va más allá de la naturaleza de la enfermedad y de su tratamiento concreto; debe continuar en una suerte de acompańamiento y orientación hacia el modo saludable de vivir. Así como el soporte informativo es fundamental, también lo es el modo de vincularse con el enfermo, con el objeto de ayudarlo a establecer un vínculo entre él y los hábitos de vida que le permitirán mantener la salud a largo plazo.

A efecto de lo que se trata de demostrar, se destacan dos de las conclusiones principales del estudio: 1 . El paciente tiene el derecho humano a la información pertinente sobre su salud (tanto respecto a la enfermedad como al tratamiento); y 2. La tendencia a establecer un marco legal específico respecto al protocolo del ejercicio de este derecho es creciente y ha alcanzado prácticamente a toda Europa. 
Así, el reconocimiento principista del derecho del paciente a la información pertinente y la tendencia hacia la formalización legal de este derecho deberían, igualmente, propiciar el ajuste de la oferta académica de las ciencias hospitalarias y afines, para incluir la gestión de la información que el profesional de la salud está obligado a compartir con el paciente.

\section{TURISMO DE SALUD}

En las últimas décadas, el desplazamiento de las personas por razones vinculadas a la salud es una tendencia que implica miles de millones de dólares en el mercado del turismo mundial. Dos factores lo determinan: uno, la inexistencia de ofertas específicas de servicios de salud de calidad en determinados rubros de la atención médica en los lugares de residencia o trabajo; dos, la diferencia significativa de precios para el mismo servicio médico en condiciones de similar calidad. Y ninguno de estos factores prescinde de la variable calidad.

Adalto Félix de Godoi, coordinador del Hospital del Corazón de Sao Paulo, en el Brasil, señala al respecto lo siguiente:

El turismo de salud, o turismo médico, ha sido testigo de un crecimiento sostenible y constante en los últimos años, y se ha convertido en uno de los servicios generadores de ingresos en los hospitales de primera línea y los considerados top del mercado. En busca de un tratamiento médico o alguna forma de recuperación de la salud, un número cada vez mayor de personas viaja a diferentes países; se convierten en lo que convencionalmente se llama "turistas de salud", y estimulan dos importantes sectores de la economía, el turismo y la salud (De Godoi 2008).
Más adelante, De Godoi agrega lo siguiente:

Aunque el término 'turismo de salud' puede traer a la mente algo relacionado exclusivamente con el turismo, se trata, en efecto, de una actividad ligada más al área de la salud. Es una tipología que ofrece la infraestructura para que un cliente de la salud viaje y satisfaga sus necesidades en ese período. Va más allá cuando complementa el período de recuperación, o pre y postratamiento, con actividades culturales, de ocio o propiamente turísticas. Así, el término adoptado no cambia la característica principal de la actividad, que es la búsqueda de la cura y no un viaje turístico, de ocio o vacaciones.

Podemos apreciar de estas afirmaciones que una de las líneas de desarrollo profesional vinculadas a la salud es la motivación turística, a partir de las ventajas competitivas de especialización o de precio/calidad que puede ofrecer un destino determinado.

Por otro lado, ofertas más típicamente turísticas como el termalismo, la talasoterapia e incluso el $s p a$, vinculadas a la salud, pero sobre todo al bienestar, son parte de una motivación especializada creciente y del desarrollo de un segmento de consumo que requiere atención específica; y que necesita del desarrollo de planta adecuada y de profesionales solventes.

Debe comprenderse que la inclusión de aquellos servicios o de aquellas competencias complementarias en la formación de los profesionales de la salud puede ser un factor diferencial de una mejor oferta académica, respecto de aquellas que no las incluyen; esto marcará un posicionamiento ventajoso de quienes lo ofrezcan en el mercado educativo. 


\section{LA ENCUESTA NACIONAL DE SATISFACCIÓN DE USUARIOS EN SALUD}

La Encuesta Nacional de Satisfacción de Usuarios en Salud del Perú (Ensusalud) es elaborada por el Instituto Nacional de Estadística e Informática (INEI) para la Superintendencia Nacional de Salud (Susalud), desde 2014. En octubre de 2015, Susalud dio a conocer los resultados del segundo estudio anual, respecto a la percepción de los usuarios (internos y externos) sobre la calidad de la atención que brindan las diversas instituciones prestadoras de servicios de salud (Ipress) a nivel nacional (INEI 2015).

La muestra de la encuesta a usuarios externos alcanzó 13670 personas mayores de 15 años y las IpRess materias de indagación fueron centros médicos del Ministerio de Salud y gobiernos regionales, de EsSalud, de servicios de sanidad de las Fuerzas Armadas y Policiales, y de clínicas del sector privado. La encuesta precisa, entre otras cosas, que la satisfacción global del usuario ha mejorado relativamente. Sin embargo, desmejoró en EsSalud, lo que debería conducir a una evaluación específica de esta entidad.

Un punto crítico general que la encuesta identifica es el tiempo promedio de espera para la atención en consulta externa: en 2014, el tiempo promedio era de 81 minutos; en 2015 fue de 104 minutos; el rango de variación va de 42 minutos en las clínicas privadas, a 148 minutos en los centros de salud del Minsa-gobiernos regionales. Otro asunto crítico es el tiempo de espera para obtener una cita médica: en la encuesta de 2014, este tiempo era de 17 días; en la de 2015 es de 18 días; el rango de variación es de 10 días en las clínicas privadas, a
19 días en los centros de salud del Minsa-gobiernos regionales y en los servicios de sanidad de las Fuerzas Armadas y Policiales (INEI 2015: 12). Hay identificado otro tema agudo: en 2014, el tiempo promedio de atención al usuario era de 14 minutos; en 2015 es de 12 minutos; el rango de variación es de 15 minutos promedio en las clínicas privadas, a 11 minutos promedio en los servicios de sanidad de las Fuerzas Armadas y Policiales y en EsSalud (INEI 2015: 208).

Podemos apreciar que estos puntos críticos están relacionados con procesos administrativos o de dedicación del profesional hacia el paciente. Así, el desarrollo de las competencias blandas de todos los participantes en la cadena del servicio de salud es fundamental para su mejora significativa; y esto supone el servicio de la hospitalidad.

\section{A MODO DE RESUMEN}

La hospitalidad es una propuesta holística, que va más allá de la curación de las enfermedades. La función curativa del hospital no es un tema exclusivo de la salud; incluye un conjunto de actitudes y servicios que deben contribuir a la colaboración del paciente en su curación. Un paciente no es un sujeto pasivo de la atención médica o curativa; es una persona cuyas expectativas están modificadas justamente por la pérdida de la salud y requiere ser tratado con excepcionalidad positiva por la misma razón.

La información pertinente es el primer derecho del paciente para guiarlo al encuentro con la recuperación de su salud y la adopción de hábitos saludables. Asimismo, el turismo de salud es una motivación de viaje creciente, que los profesionales de la medicina y de la 
salud deben estar en condiciones de atender satisfactoriamente. Aquí, la curación es solo un componente de la oferta imprescindible. El bienestar del paciente es el objetivo final.

La medición desagregada de la satisfacción (o insatisfacción) del paciente hospitalario es un aspecto fundamental de la mejora de la oferta profesional de salud. El periodo que demora para obtener una cita o el tiempo de espera para ser atendido o el periodo que dura la atención son, entre muchos otros, elementos que definen dicha satisfacción (o insatisfacción).

Este interés se inicia, o debe iniciarse, en la formación educativa profesional; y no se limita a profesiones de salud; se extiende a las ciencias administrativas, y aun a la ingeniería. La educación universitaria de los profesionales debe integrar aprendizajes y conocimientos que apunten orgánicamente a la solvencia de las necesidades de salud de los pacientes, incluida la exigencia de hospitalidad. Estas, si bien en un inicio pudieran parecer muy subjetivas, son exigencias del mercado y parte de los derechos fundamentales de las personas en una sociedad como la contemporánea.

\section{BIBLIOGRAFÍA}

\section{BERRY, L.; PARASURAMAN, A.}

1993 Marketing en las empresas de servicios. Compita mediante la calidad. Bogotá: Norma.

DE GODOI, Adalto Félix

2008 "El turismo de salud: Una visión de la hospitalidad mundial". El Hospital. Consulta: 26 de mayo de 2016. Disponible en: <http://www.elhos-
pital.com/temas/El-turismo-de-salud,-Una-vision-de-la-hospitalidad-mundial+8066855>.

CARRERA MUÑOZ, Agustín

1879 Hospitalidad y hospitales [Manuscrito]: memoria presentada por... Agustín Carrera y Muñoz para obtar (sic) al título de Doctor... Madrid: Universidad Central (Madrid), Facultad de Medicina. Consulta: 1 de agosto de 2016. Disponible en: $<$ http://alfama.sim.ucm.es/dioscorides/consulta_libro.asp?ref=b18951223\&idioma $=0$ >

\section{INEI}

2015 Encuesta Nacional de Satisfacción de Usuarios en Salud. Informe Final. Consulta: 14 de setiembre de 2016. Disponible en: <http://portales.susalud.gob.pe/ documents/11450/134899/INFORME+FINAL+-+SATISFACCI\%C3\%93N+2015/b2404ecb-0aff-4cea-87bd-acd91b8225fc>.

MOLES, Vicente Juste

s/f "Hospital, Hospitalidad y Hospedaje. Historia del Hospital de Benasque". Hospital de Benasque. Hospedería. Benasque. Consulta: 11 de agosto de 2016.

Disponible en: <http://www.llanosdelhospital.com/eng/Media/Files/ Historia-de-los-Hospitales>.

\section{VALLS CASAS, Aurora; RODRÍGUEZ} PARADA, Concepción

2008 "El derecho a la información del paciente: una aproximación legal y deontológica". BiD: textos universitaris de biblioteconomia i documentació, núm. 21 (diciembre). Consulta: 5 de julio de 2016.

Disponible en: <http://bid. ub.edu/21/vall2.htm>. 